\title{
Patient commentary: What I need to self manage my care
}

\author{
Alex Silverstein past president \\ International Diabetes Federation's Young Leaders in Diabetes Programme
}

Managing my health has, and always will be, a partnership. I had type 1 diabetes diagnosed at age 18 months and I am now 27. Along the way my diabetes has been managed in a partnership between family (mainly my mother), friends, school, college, the workplace, the charity sector, health professionals, drug companies (who provide insulin, test strips, etc), the patient community (through peer support and online resources), and, most importantly, me, the person at the centre.

As I have grown older, the responsibilities and level of involvement of each of these have changed, and I now feel confident in managing my health. These are the things that help me.

Medical devices and repeat prescriptions-I order these through an app and website installed by my general practice. I can choose which pharmacy my repeat prescription goes to or they can deliver to my house, similar to internet shopping. The app also lets me book appointments and look at my medical records when it suits me. My insulin pump has a programme to upload all my blood test results and carbohydrate and insulin intake, and I can access and analyse these at home on my laptop.

Multidisciplinary healthcare team in secondary care-I trust the team to provide me with the best advice and technology available, and the team trusts me to lead and advise them on what my care should look like.

Education-As I self manage for more than $99 \%$ of the time I need to understand how to treat my diabetes and this requires ongoing education. The first self management education I received about diabetes was a week's course with my nurse and dietitian when I was 18. I then also had a three day course for my pump. For me this was too little, too late. Healthcare professionals should provide age appropriate education for those responsible for the day to day management of their care (be that a carer or the patient themselves), at the time of diagnosis or signpost patients to appropriate education from charities or websites. I get my ongoing education, especially about the latest research, from patient communities on Twitter, Facebook, and other websites, without the need to visit health professionals. If we are to reach 385 million people with diabetes worldwide, this is the only sensible method. But patients should have access (and be directed) to approved online courses to ensure quality. Empathy - The first step to regaining control in your life after being diagnosed with a long term health condition is to recognise the normality of shock, anger, and grief that you, or your carer, will immediately feel. You then have to slowly learn what positive steps you can take, from others that have been through the same experience. If people are to manage their health successfully we must recognise as a whole system that this support is generally found within patient communities rather than in a clinic. Health professionals should therefore be able to inform people about patient communities and online resources.

Engagement-This is different from compliance. It's about finding opportunities for experiential learning and spending time to educate yourself about your condition through finding or providing peer support.

Integrated services - General practice and secondary services should be integrated and provide friendly personalised guidance, so that patients are keen and motivated to access all the avenues open to them. Luckily for me, my mother, my nurse, and my previous employers (Diabetes UK and the International Diabetes Foundation) as well as many of the fellow patient leaders I have met along the way have guided me to find and use the different services on offer.

I now feel I can manage my health independently except in a crisis. However, I don't think this is recognised: I am still requested to go to the hospital once every six months for an appointment that may not be needed, to take tests that I could access locally, and to look at healthcare data that are requested, delivered, and stored by my hospital-not by me. This is not person centred care, and health professionals should instead be focusing on patients that need more support than me.

For me the next logical steps are to create my own care plan, containing goals set and measured by me and accessible at a time and location that suits me (online or offline), and to work with the stakeholders I choose, from the list mentioned above, so that I can achieve and update my care plan. 
What I don't want is to be given routine outpatient appointments. People don't want to spend time in clinics to manage their health; they want to do it at home in a way that suits them, like their banking or shopping. This is person centered care and in some places it's already happening. But we will only get universal person centered care when we begin valuing, measuring, and responding to the things that patients really care about-and doing so in a systematic, fully integrated "whole system" way.

Competing interests: I have read and understood BMJ policy on declaration of interests and have the following interests to declare. I am an NHS employee working in patient experience and equalities across Central London, West London, Hammersmith and Fulham, Hounslow and Ealing Clinical Commissioning Groups Collaborative Clinical Commissioning Groups. I have received honorariums for presentation on the use of social media in the treatment of diabetes.

Provenance and peer review: Commissioned; not externally peer reviewed.

Cite this as: BMJ 2015;350:h248

๑ BMJ Publishing Group Ltd 2015 